Gdańsk 2020, Nr. 43

https://doi.org/10.26881/sgg.2020.43.05

Dominika Janus

Universität Gdańsk / Uniwersytet Gdański

ORCID: 0000-0001-8817-6948

\title{
Historische Text(sorten)linguistik in germanistischer Forschung in Deutschland und in Polen
}

\begin{abstract}
Das in den siebziger und achtziger Jahren formulierte Postulat 'Sprachgeschichte als Textsortengeschichte' wurde in der germanistischen Forschung in Deutschland erst in den nächsten Jahrzehnten empirisch umgesetzt. Gemeint sind hier zahlreiche Monographien sowie Sammelbände. Von diesen Arbeiten gingen Inspirationen für polnische Germanist(inn)en aus, die eine Erforschung historischer Textsorten unternahmen. Das Ziel des vorliegenden Beitrags ist es, eine Bilanz aus dem bereits Untersuchten zu ziehen und Perspektiven künftiger Forschung in diesem Bereich zu schildern.
\end{abstract}

Schlüsselwörter: Historische Text(sorten)linguistik, Sprachgeschichte, Textsortengeschichte, Analyse historischer Texte, interne oder externe Textmerkmale

Historical linguistics of text in German studies in Germany and Poland. - The postulate of 1970-ies and 1980-ies 'History of language as history of text genres' was practically used only in the following decades. A lot of monographies and collective works appeared. Those works became an inspiration for Polish Germanists who undertook research on historical genres. The aim of this article is to make the balance of the achievements in this domain and forecasting further development of this discipline.

Keywords: historical linguistics of text, history of language, history of genres, analysis of historical texts, inner and outer features of text

\section{Zielsetzung}

Das in den siebziger und achtziger Jahren formulierte Postulat 'Sprachgeschichte als Textsortengeschichte' (Schenker 1977; Steger 1984; Schank 1984), nach dem der Sprachwandel nicht als Wandel bestimmter textlicher Mittel, sondern als Wandel bestimmter Textsorten anzusehen ist (Cherubim 1980: 16), wurde in der germanistischen Forschung in Deutschland (und Österreich) erst in den nächsten Jahrzehnten empirisch umgesetzt. Gemeint sind hier Detailstudien sowie Sammelbände. Aus diesen Arbeiten gingen natürlich Inspirationen für die polnischen Germanist(inn)en, die eine Erforschung historischer Textsorten unternahmen. 
Der Beitrag setzt sich zum Ziel, eine Bilanz aus dem bereits Untersuchten zu ziehen ${ }^{1}$ und Perspektiven künftiger Forschung im Bereich der historischen Text(sorten)linguistik zu schildern. Aus diesem Grund wird versucht, einen resümierenden Einblick in den Forschungsstand sowohl in Deutschland, als auch in Polen zu geben. Zum Schluss wird aus dem Dargestellten das Fazit gezogen, wobei das besondere Augenmerk den Fragen nach Entwicklungsperspektiven der Text(sorten)linguistik gilt.

\section{Historische Text(sorten)linguistik in germanistischer Forschung in Deutschland}

Die Forderung nach einer textsortenbezogenen Fokussierung der Sprachgeschichtsschreibung und dadurch nach einer pragmatischen Orientierung der Sprachgeschichte fand ihren Ausdruck im Postulat 'Sprachgeschichte als Textsortengeschichte', das im Jahre 1977 von Walter Schenker schriftlich erhoben wurde. Der Forscher schrieb damals: „Sprachgeschichte sei, selbstverständlich eingebettet in die Sozialgeschichte, wesentlich als Textsortengeschichte zu begreifen." (Schenker 1977: 141), d. h. sie sei nicht mehr als „die summierte Geschichte der grammatischen Teilbereiche" (Schenker 1977: 147) zu betrachten. In seinem Aufsatz aus dem Jahre 1984 sah Hugo Steger die Notwendigkeit zur Erweiterung des Forschungsprogramms der Sprachgeschichte zur Kommunikationsgeschichte, indem er feststellte: „,...] die dt. Sprachgeschichte [stellt sich] (auch) dar als Geschichte der Ausgliederung und des Ausbaus von funktionalen Sprachvarietäten und Texttypen-/Gattungsinventaren, die für kommunikative Aufgaben in einer sich differenzierenden Welt speziell geeignet sind" (Steger 1984: 200). Auf den Zusammenhang von Textsortengeschichte und Sprachwandel wies auch Gerd Schank hin. Der Forscher betonte, dass mit dem Begriff der Textsorte kommunikative Handlungskontexte thematisiert werden könnten. Er präzisierte: „Im Wandel von Textsorten wird Sprachwandel als Wandel sowohl zeichenlinguistischer Elemente wie auch pragmatischer Muster greifbar" (Schank 1984: 762).

Da die oben erwähnten Beiträge vor allem konzeptionell orientiert waren, konstatierte Ingo Warnke (1995: 96) ein Jahrzehnt später, dass „der Vielzahl programmatischer Forderungen nach einer Textorientierung sprachgeschichtlicher Fragestellungen nur wenige konkrete Textanalysen gegenüberstehen.“ Der Forscher fügte hinzu: „Es scheint also, als sei zwar ein neues historiolinguistisches Paradigma eröffnet, das jedoch weder durch empirische Befunde ausreichend gedeckt noch in Frage gestellt ist" (Warnke 1995: 96). Einer der Gründe dieses Bruchs zwischen Theorie und Praxis bestehe darin, so Warnke, dass sich das Postulat 'Sprachgeschichte als Textsortengeschichte' bisher nicht genügend mit der Konzeption eines pragmatisch orientierten Verfahrens zur Analyse historischer Texte verbindet. Als Ausnahme nennt er die Untersuchung von Johannes Schwitalla (1983).

Auf konkrete Textanalysekonzepte, die den Besonderheiten der sprachhistorischen Problemstellung entsprechen, musste man bis zur Zeit um die Jahrhundertwende warten. Erst kurz vor und nach 2000 sind nämlich bedeutende Arbeiten entstanden, die Konzeptionen

1 Dazu auch in Wiktorowicz (2009: 265-273). 
eines pragmatisch orientierten Verfahrens zur Analyse historischer Texte darstellen und praktisch umsetzen. Genannt werden sollen Sammelbände (herausgegeben u. a. von Simmler 1997, 2002, 2004; Wiesinger 2007; Meier/Piirainen 2007; Schwarz/Simmler/Wich-Reif 2009; Habermann 2011; Ernst/Meier 2014; Schuster/Holftreter 2017; Simmler/Wich-Reif 2019a, 2019b) und Monographien. Die letzteren (hier nur selektiv präsentiert) können dabei je nach dem Kommunikationsbereich, zu dem die analysierten Texten gehören, eingeteilt werden. Unter den Arbeiten zu den Textsorten der Kanzlei (Bereich Institution) sollen u. a. die von Grolimund (1995), Möller (1998), Topalović (2003), Ziegler (2003), Meier (2004), Bieberstedt (2007), Lange (2008) genannt werden. Dem Bereich Wirtschaft und Handel ist die Arbeit von Tophinke (1999) zuzuordnen. Die Textsorten aus dem Bereich Technik analysiert Fleskes (1996) und Zirngibl (2003). Zum Bereich Alltag bzw. Öffentlichkeit gehören die Studien von Bendel (1998) und Hölscher (2011). Schuster (2001) und Pfefferkorn (2005) widmeten sich in ihren Monografien den Texten aus dem religiösen Bereich.

Eines der Probleme, mit dem sich die Text(sorten)linguistik, darunter die historische Text(sorten)linguistik, beschäftigt, ist die Frage danach, welche Merkmale eine Textsorte konstituieren. Obwohl heutzutage kein Zweifel mehr daran besteht, dass bei der Analyse einer Textsorte mehrere Dimensionen, d. h. sowohl textinterne, als auch textexterne Faktoren, berücksichtigt werden müssen, sind sich die Autoren der oben dargestellten Arbeiten darin nicht einig, ob die internen oder externen Textmerkmale entscheidend sind. Aus diesem Grund wird im Folgenden versucht, diejenigen Projekte kurz zu präsentieren, die sich in der Beurteilung der Rolle der textinternen und -externen Faktoren voneinander unterscheiden. Bei der Auswahl der Autoren wurde außerdem die Tatsache beachtet, welche von ihnen das Profil der historischen Text(sorten)linguistik in der germanistischen Forschung in Polen am meisten beeinflussten.

Eine eigenständige Methode, die zur Untersuchung von historischen (aber auch gegenwartssprachlichen) Textsorten dienen kann, entwickelte Franz Simmler (1988, 1990, 1997, 2007, 2009). Der Forscher geht davon aus, dass im Rahmen einer Textsorte interne und externe Merkmale verbunden sind. Die externen Faktoren können dabei bei allen historischen Überlieferungen nicht unabhängig von den internen gewonnen werden. Die internen Merkmale verweisen nämlich auf die externen und die von ihnen gebildeten externen Variablenkonstellationen. Als externe Merkmale gelten Produzent, Adressat, Ort und Zeit (Simmler 2007: 210). Zu den internen Merkmalen gehören Makrostrukturen, darunter Initiatoren, Terminatoren, Bilder mit Unterschrift, Absätze usw. (Simmler 1997: 66). Für die Analysemethode von Simmler ist daher kennzeichnend, dass man sich hier stark auf die textinternen Faktoren konzentriert, ohne dabei die anderen Dimensionen zu berücksichtigen.

Auch die Untersuchungsmethode von Andreas Bieberstedt (2007) ist auf die textinternen Faktoren ausgerichtet. Für die Analyse der volkssprachigen Testamente des 14. und 15. Jahrhunderts wird ein Vier-Ebenen-Schema angesetzt, das drei Textstrukturebenen und eine syntaktische Realisierungsebene umfasst. Zu den Textebenen gehören Makrostruktur, Basisstruktur und Substruktur. Die Makrostruktur erlaubt die Zuweisung von Textsorten zu einem übergeordneten Texttyp (z. B. die Zuordnung der Textsorte Testament zum Texttyp Urkunde). Die Basis- und Substruktur konstituieren die Architektur des Textes. Ihre Prototypikalität führt zur Entstehung unterschiedlicher Text(sorten)muster. Die vierte syntaktische Ebene 
bezieht sich auf die Mikrostruktur eines Textes, die „die konkrete syntaktisch-lexikalische Realisierung textstruktureller Einheiten" (Bieberstedt 2007: 33) bedeutet.

Für eine Integration der textinternen und textexternen Merkmale plädieren wiederum Arne Ziegler (2003) und Jörg Meier (2004). Die Forscher entwickelten nämlich ein integratives Modell einer Historischen Textlinguistik auf soziopragmatischer Grundlage, das ihnen eine Typologisierung der kanzleisprachlichen Texte ermöglicht. Meier/Ziegler (2004) nehmen eine Makroklassifizierung nach den textexternen Merkmalen zum Ausgangspunkt, d. h. sie nennen die soziopragmatischen Faktoren, zu denen die Öffentlichkeiten, Kommunikationsbereiche, Handlungsbereiche sowie Funktionsbereiche gehören. Erst dann gehen sie zu einer Klassifikation der analysierten Texte nach den textinternen Merkmalen über. Vermittelt werden hier die Textstrukturen, d. h. eine Makro- und Mikrostruktur. Der Begriff Makrostruktur wird hier als „Globalstruktur des Textes“verstanden und zerfällt in zwei Kategorien Textarchitektur und Textkomposition (Ziegler 2003: 69-70). Die Architektur bezieht sich auf „die äußere Gestaltungsweise“, die Komposition dagegen auf „die inhaltliche Strukturierung des Textes" (Ziegler 2003: 70-71). Es werden hier einerseits horizontale, andererseits vertikale Textarchitekturen unterschieden (Ziegler 2003: 249). Die Mikrostruktur ist durch die topikalisch-rhematischen Verknüpfungen des Textes und deren Propositionen bestimmt (Ziegler 2003: 71).

Als ein Neuansatz für die sprachhistorische Forschung ist auch das Konzept von Oliver Pfefferkorn (2005) zu betrachten. Statt eines Rasters, in das die textinternen und textexternen Merkmale isoliert eingeordnet werden, schlägt der Forscher ein Bündel von fünf Textgestaltungsprinzipien vor. Das Mehr dieser Textgestaltungskriterien im Gegensatz zu einer von außen angelegten Matrix erhellt er folgendermaßen:

„Der Vorteil von Textgestaltungsprinzipien als Differenzierungskriterien im Gegensatz zu einzelnen sprachinternen und -externen Merkmalen besteht darin, dass mit ihnen die Texte und Textsorten in ihrer Komplexität in den Blick kommen und nicht einzelne Merkmale isoliert werden. Diese Textgestaltungsprinzipien schlagen sich in den Textsorten zwar in einzelnen Merkmalen nieder, zugleich erfassen sie aber das Zusammenwirken dieser Merkmale, die ganz verschiedenen Ebenen entstammen können. Hinzu kommt, dass diese Textgestaltungskriterien nicht relationslos nebeneinander stehen; zwischen ihnen existiert ein engmaschiges Netz ganz verschiedener Beziehungen [...]. Sie unterstützen sich gegenseitig in ihrer Funktion für den gesamten Text" (Pfefferkorn 2005: 376).

Zu fünf Textgestaltungsprinzipien, die Pfefferkorn (2005: 161-163) als konstitutiv für den gesamten Textbereich der Erbauungsliteratur betrachtet, gehören: Rhetorik, Polyfunktionalität und Mehrfachadressierung, Meditation, Intertextualität und Mystik. Diese Prinzipien ermöglichen die Erbauungsliteratur von anderen Textbereichen (z. B. den Textsorten der Kanzlei) abzugrenzen (Pfefferkorn 2005: 199-205).

\section{Historische Text(sorten)linguistik in germanistischer Forschung in Polen}

Die Untersuchung der historischen Texte im polnischen Raum konzentriert sich zu einem großen Teil auf die Analyse der graphematisch-phonologischen (u. a. Wiktorowicz 1981; 
Grabarek 1984; Kaleta-Wojtasik 2004; Biszczanik 2013; Biaduń-Grabarek/Firyn (Hg.) 2017) oder grammatischen Besonderheiten (u. a. Firyn 2012; Biszczanik 2018).

Die Auseinandersetzung mit diachronen Textsorten aus der Perspektive der Textlinguistik erfreut sich bei den polnischen Germanist(inn)en eher geringer Beliebtheit. Unter den Forschungszentren, die sich mit dieser Thematik befassen, ist das Warschauer Team um Józef Wiktorowicz (u. a. Anna Just, Waldemar Czachur, Ireneusz Gaworski, Małgorzata Bralska) am aktivsten. Im Mittelpunkt des Interesses von Wiktorowicz stehen die Textsorten der Krakauer Kanzlei (u. a. Testament, Schuldbrief, Stadtordnung). In der Diskussion, ob sich die Textsorten anhand eines textinternen oder textexternen Merkmalskatalogs bestimmen lassen, vertritt der Forscher die Meinung, dass zunächst von den textexternen Faktoren auszugehen sei. Wiktorowicz betont dabei, dass „die Sprachgeschichte und auch die Geschichte der Textsorten in hohem Maße durch soziale Faktoren determiniert wird" (Wiktorowicz 2011²: 160). Aus diesem Grund betrachtet er die Textsortengeschichte als „Reflex der Sozialgeschichte“ und die Textsorten als „Produkte des sozialen Handels" (Wiktorowicz 2011: 129). Der Forscher stellt in diesem Kontext fest:

„In sprachhistorischer Perspektive [...] werden die Texte primär nicht produziert, um jemanden über etwas zu informieren. Zunächst wird eine soziale Handlung vollzogen, die für andere soziale Partner von großer Relevanz ist. Daher muss sie schriftlich fixiert werden, damit die zeitgenössischen und zukünftigen Mitglieder einer Sprachgemeinschaft die getroffenen Entscheidungen einer sozialen Gruppe oder eines einzelnen sozialen Partners zur Kenntnis nehmen und dementsprechend handeln" (Wiktorowicz 2011: 128).

Die vollzogene soziale Handlung gilt daher als Grundkriterium für die Differenzierung von Textsorten (Wiktorowicz 2011: 131).

Texte als Handlungen zu beschreiben ist das Ziel der Studie von Waldemar Czachur (2007a) zu Vereinssatzungen im 19. Jahrhundert. Der Forscher realisiert es mittels einer kombinierten bzw. holistischen Untersuchungsmatrix, im Rahmen derer der handlungsorientierte Ansatz zur Textsortenanalyse begründet wird. Seine Methode erklärt er folgendermaßen: „Ausgehend von dem konstruierten Textmuster schreitet die Analyse vom Allgemeinen (Gesamthandlung) zum Spezifischen (sprachliche Handlungsstrukturen und Formulierungsmuster)" (Czachur 2007a: 98). Im ersten Schritt, d. h. in der Beschreibung der Gesamthandlung, wird also die Erfassung des Kontextes, in dem der Text entsteht und verwendet wird, angestrebt. Als zweiter Schritt der Untersuchung betrachtet Czachur die Analyse der Mittel zur Erreichung der pragmatischen Ziele.

Anna Just (2014) widmete ihre Studie der Edition und Analyse von Liegnitzer Briefen aus den Jahren 1546-1678. Als Beschreibungsmodell wurde das von Ziegler/Meier entwickelte Modell der Historischen Textlinguistik auf soziopragmatischer Grundlage herangezogen, weil es erlaubt, „die Mehrdimensionalität einer Auseinandersetzung mit historischen Texten nachzuvollziehen" (Just 2014: 102). Nach der Darstellung der historisch-soziopragmatischen und historisch-textlinguistischen Einbettung der Liegnitzer Briefe geht die Forscherin zur

2 Es handelt sich dabei um eine Sammlung der Beiträge von Wiktorowicz, die in den früheren Jahren in verschiedenen Sammelbänden veröffentlicht wurden. 
Analyse ihrer Textstrukturen über. Sie analysiert erstens horizontale Textarchitekturen, zweitens Makrostrukturen (die strukturellen Hauptbestände).

Unter anderen Forschungszentren, in denen Arbeiten zur historischen Text(sorten)linguistik entstanden sind, ist vor allem Krakau und Breslau zu nennen. Die Dekrete des Krakauer Oberhofs (1456-1504) stehen im Zentrum der Untersuchung von Krystyna Waligóra (2009). Das Ziel ihrer Arbeit ist die Feststellung textinterner und textexterner Merkmale und auf Grund von Oppositionen die Ermittlung von Textsorten und Textallianzen. Die Forscherin bedient sich dabei der Methodik und des Begriffsapparats zur Textsortenanalyse von Simmler. Der Breslauer Beitrag der polnischen Germanistik zur Erforschung der historischen Textsorten ist die Monographie von Józef Jarosz (2017), die den Sprachwandelprozessen auf der Ebene der Texte und ihrer Muster am Beispiel der Textsorte Grabinschrift (1780-2015) gewidmet ist. Der Autor benutzt ein Instrumentarium einer holistischen Textanalyse, um „[den] Entstehungskontext, die damit zusammenhängenden Textfunktionen sowie die Textmerkmale auf allen relevanten Ebenen der Sprachbetrachtung einschließlich der textsemiotischen" (Jarosz 2017: 117) beschreiben zu können.

An dieser Stelle sind nicht nur Monographien zu nennen, sondern auch kleinere Beiträge der polnischen Germanist(inn)en zu Fragen der historischen Text(sorten)linguistik, u. a. von Kaleta-Wojtasik (2001), Weigt (u. a. 2005, 2009, 2013) und Dąbrowska-Burkhardt (u. a. 2016a, 2016b, 2017).

\section{Fazit}

Das Thema des Bandes „30 Jahre germanistische Forschung in Polen und Deutschland: 1989-2019“ bietet eine Gelegenheit zum resümierenden Einblick in den Forschungsstand zur historischen Text(sorten)linguistik in den beiden Nachbarländern sowie zur Darstellung der Entwicklungsperspektiven für diese Subdisziplin.

Aus dem bereits Dargestellten resultiert eindeutig, dass sich das Postulat 'Sprachgeschichte als Textsortengeschichte', das die zentrale Funktion der historischen Textlinguistik für die Sprachgeschichtsschreibung deutlich macht, erst unter den deutschen (und österreichischen), dann auch den polnischen Germanist(inn)en durchgesetzt hat. Im Rahmen dieses neuen Paradigmas entstand (allerdings mit einer gewissen Verzögerung) - erst in Deutschland (und Österreich) - eine Reihe von Sammelbänden und Detailstudien, in denen die Konzeptionen eines pragmatisch orientierten Verfahrens zur Analyse historischer Texte mit Versuchen ihrer Verwendung effizient kombiniert werden. Die polnische Auslandsgermanistik (besonders das Warschauer Forschungszentrum) reagierte auf diese Neuerungen, indem sie ihre eigene Forschung zu historischen Textsorten unternahm.

Wenn es sich um Perspektiven und Desiderate handelt, muss vor allem auf die Notwendigkeit der Integration der historischen Text(sorten)linguistik in den Rahmen einer gegenwärtig immer noch dominant synchron orientierten Text(sorten)linguistik hingewiesen werden. Die historische Textlinguistik muss dabei jedoch ihre Spezifik beibehalten. Wie Wiktorowicz (2011: 127) betont, sind die theoretischen Ansätze der synchronen Textsortenanalyse für eine historische Textsortenanalyse wenig geeignet. Als nicht geeignet ist 
in erster Linie die Übernahme des meist textinternen Merkmalskatalogs für die historischen Textsortenklassifikation zu betrachten. Für diachrone Textsortenforschung sind indessen die textexternen Merkmale entscheidend.

Es sollten weitere pragmatisch orientierte Studien angestrebt werden, die konkreten historischen Textsorten gewidmet werden. Texte bzw. Textsorten sind hier in den Verwendungskontexten zu untersuchen. Die Analyse von Textsorten sollte Texte in ihren komplexen thematischfunktionalen Zusammenhängen umfassen. Das Ziel der historischen Text(sorten)linguistik sollte auch darin bestehen, Texte bzw. Textsorten nicht isoliert, sondern in ihrem funktionalen Zusammenwirken (vgl. Textsorten-Intertextualität) zu betrachten. ${ }^{3}$ Diese Initiativen sollen nicht nur die Inlandsgermanistik anregen. Auch polnische Germanist(inn)en haben hier ihre Aufgabe zu erfüllen. In vielen Forschungsbibliotheken und Archiven des heutigen polnischen Raumes werden deutschsprachige Textsammlungen aufbewahrt, die zum Untersuchungsgegenstand der historischen Text(sorten)linguistik werden könnten und sollten. ${ }^{4}$

\section{Literatur}

Bendel, Sylvia (1998): Werbeanzeigen von 1622-1798. Entstehung und Entwicklung einer Textsorte. Tübingen: Niemeyer.

Biaduń-Grabarek, Hanna / Firyn, Sylwia (Hg.) (2017): Sprache der deutschsprachigen Kanzleien in der frühneuhochdeutschen Zeit im südlichen Ostseeraum. Teil 1: Phonologische und graphematische Ebene. Frankfurt a. M.: Peter Lang.

Bieberstedt, Andreas (2007): Textstruktur - Textstrukturvariation - Textstrukturmuster: Lübecker mittelniederdeutsche Testamente des 14. und 15. Jahrbunderts. Wien: Praesens.

Biszczanik, Marek (2013): Die ältesten Stadtbücher von Sprottau/Szprotawa: ein Beitrag zur Erforschung des frühneuhochdeutschen in Niederschlesien. Zielona Góra: Oficyna Wydawnicza Uniwersytetu Zielonogórskiego.

Biszczanik, Marek (2018): Sprachwandel im Bereich der verbalen Morphosyntax: eine korpusgestützte Untersuchung am Handschriftenmaterial der Schweidnitzer Kanzlei im 13.-16. Jahrhundert. Zielona Góra: Oficyna Wydawnicza Uniwersytetu Zielonogórskiego.

Cherubim, Dieter (1980): Zum Programm einer historischen Sprachpragmatik. In: Horst Sitta (Hg.): Ansätze zu einer pragmatischen Sprachgeschichte. Tübingen: Niemeyer, 3-21.

Czachur, Waldemar (2007a): Textmuster im Wandel. Ein Beitrag zur textlinguistischen Erforschung der Vereinssatzungen im 19. Jahrbundert. Wrocław, Dresden: Atut.

Czachur, Waldemar (2007b): Zur diachronen Textsortenlinguistik. In: Studia Germanica Resoviensia 5, 243-252.

Dąbrowska-Burkhardt, Jarochna (2016a): Die Textsorte ‘Stammbuch' als Vorgänger von „Facebook“. Eine linguistische Analyse der Stammbucheinträge aus dem 18. Jh. am Beispiel eines Grünberger album amicorum. In: Ewa Żebrowska, Magdalena Olpińska-Szkiełko, Magdalena Latkowska (Hg.): Zwischen Kontinuität und Modernität: Metawissenschaftliche und wissenschaftliche Erkenntnisse der

\footnotetext{
3 Mehr zu diesen Postulaten der diachronen Text(sorten)linguistik in Czachur (2007b: 245).

4 Einen Beitrag der polnischen Germanistik zur Forschung der historischen Textsorten sollte das Projekt der Autorin dieses Beitrags: „Danziger Leichenpredigten (1586-1746) aus textlinguistischer Sicht“ leisten.
} 
germanistischen Forschung in Polen. Warszawa: Wissenschaftliche Beiträge des Verbandes Polnischer Germanisten, 85-93.

Dąbrowska-Burkhardt, Jarochna (2016b): Stereotype Aussagemuster und ihre semantische Analyse anhand frühneuzeitlicher Hexenverhörprotokolle aus Grünberg in Niederschlesien. In: Paweł Bąk, Bogusława Rolek (Hg.): Vom Wort zum Gebrauch: Wortbedeutung und ihre Eingebundenheit in Diskurse. Frankfurt/M.: Lang, 129-142.

Dąbrowska-Burkhardt, Jarochna (2017): Multimodalität in historischen Texten. Ein Beitrag zur historischen Textsemiotik am Beispiel eines Grünberger Stammbuchs aus dem 18. Jahrhundert. In: Zofia Bilut-Homplewicz, Anna Hanus, Agnieszka Mac (Hg.): Medienlinguistik und interdisziplinäre Forschung I: Textsortenfragen im medialen Umfeld. Frankfurt/M.: Lang, 225-241.

Ernst, Peter / Meier, Jörg (Hg.) (2014): Kontinuitäten und Neuerungen in Textsorten- und Textallianztraditionen vom 13. bis zum 18. Jahrhundert. Berlin: Weidler.

Firyn, Sylwia (2012): Junktoren im Text der Protokolle des Generallandtags von Preussen Königlichen Anteils aus den Jahren 1526-1528. Frankfurt/M.: Lang.

Fleskes, Gabriele (1996): Untersuchungen zur Textsortengeschichte im 19. Jahrhundert. Am Beispiel der ersten deutschen Eisenbahnen. Tübingen: Niemeyer.

Grabarek, Józef (1984): Die Sprache des Schöffenbuches der Alten Stadt Toruń. Rzeszów: Wydawnictwo Wyższej Szkoły Pedagogicznej.

Grolimund, Christoph (1995): Die Briefe der Stadt Basel im 15. Jahrhundert. Ein textlinguistischer Beitrag zur historischen Stadtsprache Basels. Tübingen: Francke.

Habermann, Mechthild (Hg.) (2011): Textsortentypologien und Textallianzen des 13. und 14. Jahrbunderts. Berlin: Weidler.

Hölscher, Sandra (2011): Familienanzeigen. Zur Geschichte der Textsorten Geburts-, Verbindung-und Todesanzeige, ibrer Varianten und Strukturen in ausgewählten regionalen und überregionalen Tageszeitungen von 1790 bis 2002. Berlin: Weidler.

Jarosz, Józef (2017): Grabinschrift - eine Textsorte im Wandel. Eine diachrone Studie am deutschen epigrafischen Material 1780-2015. Wrocław, Dresden: Atut.

Just, Anna (2014): Schreiben und Rescripte von Frauen und Prinzessinen aus dem Liegnitz(er) Fürsten Hause (1546-1678). Edition sowie eine historisch-soziopragmatische und historisch-textlinguistische Skizze. Frankfurt/M.: Lang.

Kaleta-Wojtasik, Sławomira (2001): Mittelalterliches Testament als Textsorte. Versuch einer Untersuchung anhand deutschsprachiger Testamente der Krakauer Bürger aus dem XV. Jahrhundert. In: Aleksander Schwarz, Laure Abplanalp Luscher (Hg.): Textallianzen am Schnittpunkt der germanistischen Disziplinen. Bern: Lang, 259-272.

Kaleta-Wojtasik, Sławomira (2004): Graphematische Untersuchungen zum Codex Picturatus von Balthasar Behem. Kraków: Wydawnictwo Uniwersytetu Jagiellońskiego.

Lange, Maria B. (2008): Sprachnormen im Spannungsfeld schriftsprachlicher Theorie und Praxis. Die Protokolle der Comerzdeputation Hamburg im 17. Jahrhundert. Berlin: de Gruyter.

Meier, Jörg (2004): Städtische Kommunikation in der Frühen Neuzeit. Historische Soziopragmatik und Historische Textlinguistik. Frankfurt/M.: Lang.

Meier, Jörg / Piirainen, Ilpo Tapani (Hg.) (2007): Studien zu Textsorten und Textallianzen um 1500. Berlin: Weidler. 
Meier, Jörg / Ziegler, Arne (2004): Textsorten und Textallianzen in städtischen Kanzleien. In: Franz Simmler (Hg.): Textsortentypologien und Textallianzen von der Mitte des 15. bis zur Mitte des 16. Jahrbunderts. Berlin: Weidler, 129-166.

Möller, Robert (1998): Regionale Schreibsprachen im überregionalen Schriftverkehr. Empfängerorientierung in den Briefen des Kölner Rates im 15. Jahrhundert. Köln: Böhlau.

Pfefferkorn, Oliver (2005): „Übung der Gottseligkeit“. Die Textsorten Predigt, Andacht und Gebet im deutschen Protestantismus des späten 16. und des 17. Jahrhunderts. Frankfurt/M.: Lang.

Schank, Gerd (1984): Ansätze zu einer Theorie des Sprachwandels auf der Grundlage von Textsorten. In: Werner Besch, Oskar Reichmann, Stefan Sonderegger (Hg.): Sprachgeschichte. Ein Handbuch zur Geschichte der deutschen Sprache. Bd. 1. Berlin: de Gruyter, 761-768.

Schenker, Walter (1977): Plädoyer für eine Sprachgeschichte als Textsortengeschichte. Dargestellt am Paradigma von Telefon, Radio, Fernsehen. In: Deutsche Sprache 2, 141-148.

Schuster, Britt-Marie (2001): Die Verständlichkeit von frühreformatorischen Flugschriften. Eine Studie zukommunikationswirksamen Faktoren der Textgestaltung. Hildesheim, Zürich, New York: Georg Olms.

Schuster, Britt-Marie / Holftreter, Susan (Hg.) (2017): Textsortenwandel vom 9. bis zum 19. Jahrhundert. Berlin: Weidler.

Schwarz, Alexander / Simmler, Franz / Wich-Reif, Claudia (Hg.) (2009): Textsorten und Textallianzen um 1500. Handbuch Teil 1: Literarische und religiöse Textsorten und Textallianzen um 1500. Berlin: Weidler.

Schwitalla, Johannes (1983): Deutsche Flugschriften 1460-1525. Textsortengeschichtliche Studien. Tübingen: Niemeyer.

Simmler, Franz (1985/86, recte 1988): Makrostrukturen in lateinischen und deutschen Textüberlieferungen der Regula Benedicti. In: Regulae Benedicti Studia. 14/15, 213-305.

Simmler, Franz (1990): Makrostrukturelle Veränderungen in der Tradition des frühneuhochdeutschen Prosaromans. In: Werner Besch (Hg.): Deutsche Sprachgeschichte. Grundlagen, Methoden, Perspektiven. Festschrift für Johannes Erben zum 65. Geburtstag. Frankfurt/M.: Lang, 187-202.

Simmler, Franz (1997): Die informationsorientierten Textsorten und ihre Varianten in der Fußballberichterstattung des «kicker sportmagazins». In: Franz Simmler (Hg): Textsorten und Textsortentraditionen. Bern: Lang, 63-144.

Simmler, Franz (2007): Zur Rolle von externen und internen Merkmalen bei der Textsortentypologie der Diatessaron- und Leben Jesu-Tradition des 16. Jahrhunderts. In: Peter Wiesinger (Hg.): Textsorten und Textallianzen vom 16. bis zum 18. Jahrhundert. Berlin: Weidler, 209-236.

Simmler, Franz (2009): Theoretische Grundlagen zur Ermittlung von Textsorten und Textallianzen und zur Reichweite des Textbegriffs. In: Alexander Schwarz, Franz Simmler, Claudia WichReif (Hg.): Textsorten und Textallianzen um 1500. Handbuch Teil 1: Literarische und religiöse Textsorten und Textallianzen um 1500. Berlin: Weidler, 11-21.

Simmler, Franz (Hg.) (1997): Textsorten und Textsortentraditionen. Bern: Lang.

Simmler, Franz (Hg.) (2002): Textsorten deutscher Prosa vom 12./13. bis 18. Jahrhundert und ibre Merkmale. Bern: Lang.

Simmler, Franz (Hg.) (2004): Textsortentypologien und Textallianzen von der Mitte des 15. bis zur Mitte des 16. Jahrbunderts. Berlin: Weidler. 
Simmler, Franz / Wich-Reif, Claudia (Hg.) (2019a): Textsorten und Textallianzen um 1500. Handbuch Teil 2, Band 1: Historiographische und rechtsgeschichtliche Textsorten und Textallianzen um 1500. Berlin: Weidler.

Simmler, Franz / Wich-Reif, Claudia (Hg.) (2019b): Textsorten und Textallianzen um 1500. Handbuch Teil 2, Band 2: Textsortengruppen der Ordnungen in der Rechts-, Verwaltungs-und Geschäftspraxis um 1500. Berlin: Weidler.

Steger, Hugo (1984): Sprachgeschichte als Geschichte der Textsorten/Texttypen und ihrer kommunikativen Bezugsbereiche. In: Werner Besch, Oskar Reichmann, Stefan Sonderegger (Hg.): Sprachgeschichte. Ein Handbuch zur Geschichte der deutschen Sprache. Bd. 1. Berlin: de Gruyter, 186-204.

Topalović, Elvira (2003): Sprachwandel - Textsorte - Dialogstruktur. Zu Verhörprotokollen aus Hexenprozessen des 17. Jahrhunderts. Trier: WVT Wissenschaftlicher Verlag Trier.

Tophinke, Doris (1999): Handelstexte. Zu Textualität und Typik kaufmännischer Rechnungsbücher im Hanseraum des 14. und 15. Jahrbunderts. Tübingen: Narr.

Waligóra, Krystyna (2009): Die Dekrete des Krakauer Oberhofs (1456-1504) als Textallianzen und Textsorten. Kraków: Wydawnictwo Uniwersytetu Jagiellońskiego.

Warnke, Ingo (1995): Typologische Aufgaben der historischen Textlinguistik. In: Linguistica. 35/1, 95-121.

Weigt, Zenon (2005): Einblicke in die Anzeigen der „Lodzer Zeitung”. In: Jörg Riecke, Britt-Marie Schuster (Hg.): Deutschsprachige Zeitungen in Mittel-und Osteuropa. Sprachliche Gestalt, historische Einbettung und kulturelle Traditionen. Berlin: Weidler, 267-278.

Weigt, Zenon (2009): Die Neue Lodzer Zeitung - ein Einblick in ihre Anzeigen. In: Waldemar Czachur, Marta Czyżewska, Agnieszka Frączek (Hg.): Wort und Text. Bestandsaufnahme und Perspektiven. Warszawa: Instytut Germanistyki Uniwersytetu Warszawskiego, 33-40.

Weigt, Zenon (2013): Die Textsorte Anzeige in der Lodzer Zeitung. In: Maria Katarzyna Lasatowicz, Andrea Rudolph (Hg.): Corpora und Canones. Schlesien und andere Räume in Sprache, Literatur und Wissenschaft. Berlin: Trafo, 351-363.

Wiesinger, Peter (Hg.) (2007): Textsorten und Textallianzen vom 16. bis zum 18. Jabrhundert. Berlin: Weidler.

Wiktorowicz, Józef (1981): System fonologiczny jezzyka niemieckiego ksiag miejskich Krakowa w XVI wieku [Das phonologische System des Deutschen in den Stadtbüchern von Krakau des 16. Jh.]. Warszawa: Wydawnictwo Uniwersytetu Warszawskiego.

Wiktorowicz, Józef (2009): Niemieckojęzyczne badania nad historycznymi gatunkami tekstu [Deutschsprachige Untersuchungen zu historischen Textsorten]. In: Zofia Bilut-Homplewicz, Waldemar Czachur, Marta Smykała (Hg.): Lingwistyka tekstu w Niemczech. Pojęcia, problemy, perspektywy. Wrocław: Atut, 265-273.

Wiktorowicz, Józef (2011): Krakauer Kanzleisprache: Forschungsperspektiven und Analysemethoden. Warszawa: Zakład Graficzny UW.

Ziegler, Arne (2003): Städtische Kommunikationspraxis im Spätmittelalter: historische Soziopragmatik und historische Textlinguistik. Berlin: Weidler.

Zirngibl, Micaëla (2003): Die fachliche Textsorte Bedienungsanleitung. Sprachliche Untersuchung zu ihrer historischen Entwicklung. Frankfurt/M.: Lang. 